\title{
Probabilistic methodology for reliability assessment of electronic packages
}

\author{
H. HAMDANI ${ }^{1}$, B. RADI ${ }^{2}$, A. EL HAMI ${ }^{3}$
}

\section{LMN, Normandie Univ, INSA Rouen, 76000 Rouen, France \& LIMII, FST Settat, BP: 577, Route de Casa, Settat, hamid.hamdani@insa-rouen.fr}

2. LIMII, FST Settat, BP: 577, Route de Casa, Settat, Morocco, bouchaib.radi@yahoo.fr

3. LMN, Normandie Univ, INSA Rouen, 76000 Rouen, France, abdelkhalak.elhami@insa-rouen.fr

\begin{abstract}
:
In the mechatronic devices, the finite element analyses are the most used method to determine time-dependent solder joint fatigue response under accelerated temperature cycling conditions, the deterministic analyses are the most used methods. However, the design variables show variability and randomness which will affect the lifetime prediction quality. This paper focuses on solder joint reliability in tape-based chip-scale packages(CSP) with the consideration of uncertainties in material parameters.
\end{abstract}

Keywords: Chip-Scale Packages ; Finite-element analysis ; Kriging metamodel ; Monte-Carlo ; Solder joint

\section{Introduction}

Currently, the chip-scale packaging technologies are widely used in electronic products, because of the increasing demand for smaller and more portable electronic devices. Specifically, in a CSP, the package footprint is less than $20 \%$ of the size of the die in which it inserted [1]. Over the traditional full-sized ball grid array (BGA), the main advantage of such package is that it saves a significant space. In this work, the tape based CSP type was studied.

However in the engineering application, the analyst is more interested in the prediction of cycles to failure i.e. the number of cycles the package can resist before failure. Consequently a combination of finite element methods with a thermal fatigue model is required[3]. Darveaux's, classified in the energy-based approach, is most popular because of the facility of its implementation. In Darveaux's methodology using FEM, the number of cycles to initiate a crack, and the number of cycles for the crack to propagate across a solder joints diameter are expressed as a function of the strain energy density accumulated per cycle during thermal cycling.

In order to predict reliability of CSP, the used simulation tools are based on deterministic approaches which does not take into account the variability and random- ness of input parameters. Consequently the Monte Carlo simulation (MCS) is widely used for uncertainty analysis. However, the complex nonlinear finite element analysis take more time to determine the time-dependent solder joint fatigue response, which makes the MCS application very expensive in term of computation. In order to efficiently overcome the computational cost, metamodel based probabilistic method is developed an applied to evaluate the reliability of CSP. The proposed method combine metamodel[2], MCS and FEM simulation.

\section{Description of the studied system}

The device analyzed is a $13 \times 13 \mathrm{~mm}$ CSP. This device is composed of 225-ball ( $15 \times 15$ full ball matrix), with $0.80 \mathrm{~mm}$ pitch and a die size which was measured at $8.24 \times 9.12 \mathrm{~mm}$.

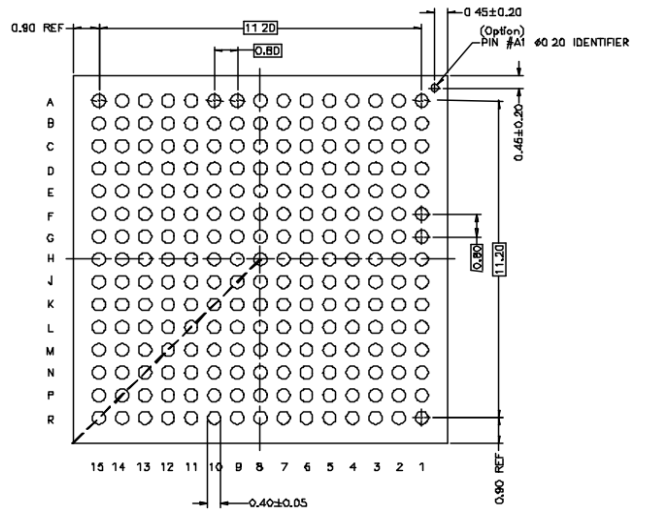

Figure 1: Package outline drawing

\section{3 probabilistic reliability analysis}

The uncertainty analysis is performed to describe the range of possible outputs depending on the uncertainty of the inputs. Monte Carlo simulation classified in the probabilistic methods is widely used for uncertainty analysis. However, to get more accurate results, MCS requires a large number of inputs samples. For the thermomechanical simulation of CSP, the non-linear transient finite element makes the simulation computationally expensive. Consequently, the classic MCS becomes impractical. For 
this reason, the metamodel based probabilistic method is proposed in order to overcome the deficiency of computational cost. This method aims to combine the metamodeling techniques with Monte Carlo simulation to perform the uncertainty analysis with high efficiency.

Kriging metamodel is a powerful tool to interpolate deterministic noise-free data [2]. It has become a standard method for constructing metamodels for computer experiments. Kriging assumes that the function to approximate is a realization of a Gaussian process denoted by $Y(x)$, which is given as :

$$
Y(x)=h(x)+Z(x)
$$

where $h(x)$ is the mean of the process, and Z(x) is a Gaussian process with zero mean.

\subsection{FEM simulation of CSP}

\subsubsection{Solder Balls finite element model}

For the purpose of avoiding tedious computation due to the complexity of physics which includes this kind of nonlinear finite element analysis, only the diagonal slice of the studied package was developed [1]. The figure 2] and 3 shows respectively, the perspective view of the meshed $3 \mathrm{D}$ diagonal slice model and the detail of the modeled solder ball.

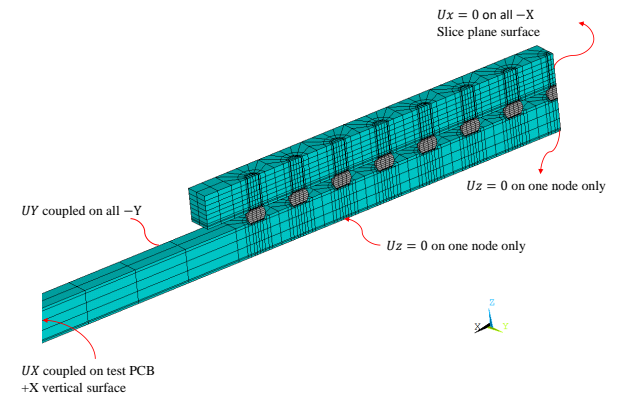

Figure 2: Boundary constraints applied to a typical slice model.

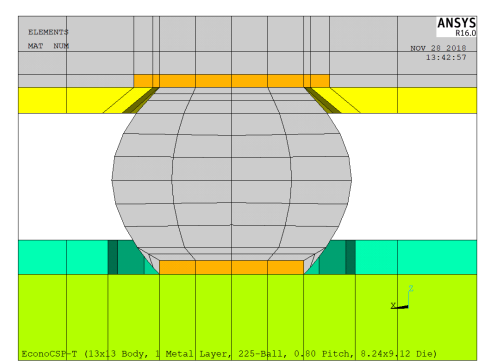

Figure 3: Modeled ball for Chip-Scale package.

\subsubsection{Material Properties}

In Zahn's paper [1] a set of eight configuration was analyzed. In this study proposed method is implemented for the first configuration where several material properties were incorporated which varies from plastic and elastic, linear and non-linear, dependent or independent of material properties on time and temperature[1]. For solder joint materials, the development of plastic strains is dependent on the rate of loading. Darveaux [1] has modified the constants in Anand's constitutive model in order to take into consideration both time independent and timedependent phenomenon. The modified Anand constants was activated for the solder ball material of studied CSP.

\subsubsection{Fatigue Life Prediction Model}

Darveaux [1] was able to establish two equations along with four crack growth correlation constants (K1 through $\mathrm{K} 4$ ), where the finite element simulation results can be used to calculate the crack propagation rate per thermal cycle and the number of cycles to crack initiation. The equations for the calculation of thermal cycles to crack initiation "No", the crack propagation rate per thermal cycle "da/dN", and the characteristic solder joint fatigue life " $\alpha$ " (number of cycles to $63.2 \%$ population failure) are shown below as (2), (3), and (4) respectively.

$$
\begin{aligned}
N_{0} & =K 1\left(\Delta W_{\text {ave }}\right)^{K 2} \\
\frac{d a}{d N} & =K 3\left(\Delta W_{\text {ave }}\right)^{K 4} \\
\alpha & =N_{0}+\frac{a}{d a / d N}
\end{aligned}
$$

Where " $\Delta W_{\text {ave }}$ is the element volumetric average of the stabilized change in plastic work within the controlled eutectic solder element thickness.

\subsubsection{Numerical results}

Once the modeling and the Ansys solution setup was completed, Accelerated thermal cycling, recommended by JEDEC standards [4], are applied as thermal load in finite element analysis. The finite element simulation provide the viscoplastic strain energy at printed circuit board solder joints and the package substrate. This viscoplastic strain energy is used to calculate solder balls characteristic life through Darveaux's crack growth rate model. Table 1 shows the details of the deterministic simulation results. 


\subsection{MCS based Metamodel}

The Monte Carlo simulation was used $10^{5}$ sample points to perform the proposed metamodel based probabilistic method. A normal probability law with $0.2 \%$ as coefficient of variation is used to introduce the dispersion of material parameters. The probability density of the characteristic life time is shown in figure 4 and 4 . To fit probability distribution, the chi-square goodness-of-fit test is used, the result shows that the Lognormal and gamma laws can be used as best fitting probability distribution for both cases.

\begin{tabular}{|l|l|l|}
\hline $\begin{array}{l}\text { Data Descrip- } \\
\text { tion }\end{array}$ & \multicolumn{2}{|c|}{ Results Solder Joint } \\
\hline & Ball/Substrate & Ball/Test Board \\
\hline $\begin{array}{l}\text { Failure Joint } \\
\text { (From Center) }\end{array}$ & 7 & 8 \\
\hline $\begin{array}{l}\text { Delta Plastic } \\
\text { Work/Cycle } \\
\text { (MPa) }\end{array}$ & 0.4987 & 0.2034 \\
\hline $\begin{array}{l}\text { Crack Initiation } \\
\text { (cycles) }\end{array}$ & 33 & 131 \\
\hline $\begin{array}{l}\text { Crack Growth } \\
\text { Rate (mm/cycle) }\end{array}$ & $0.9883 \times 10^{-03}$ & $0.4104 \times 10^{-03}$ \\
\hline $\begin{array}{l}\text { Crack Propaga- } \\
\text { tion (cycles) }\end{array}$ & 283 & 682 \\
\hline $\begin{array}{l}\text { Characteristic } \\
\text { Life (cycles) }\end{array}$ & 317 & 813 \\
\hline
\end{tabular}

Table 1: Simulation results of Chip-Scale Package.

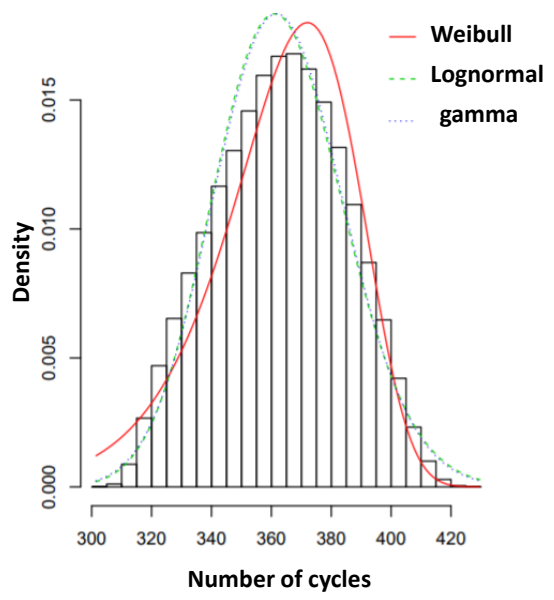

Figure 4: Probability density function of the characteristic life time of the Ball/Substrate Solder joint.

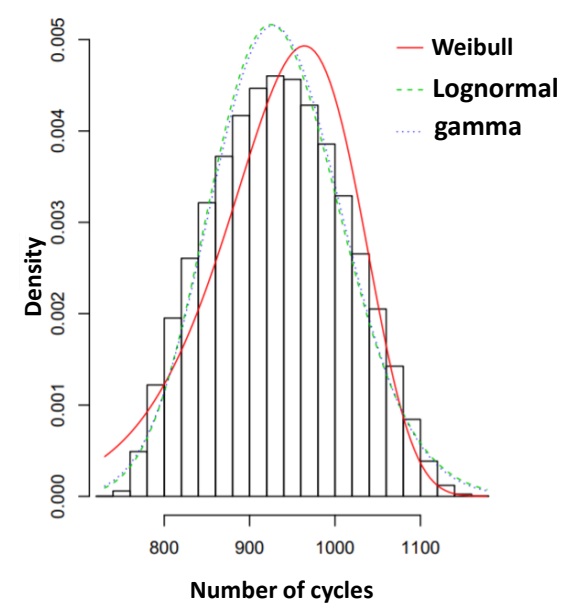

Figure 5: Probability density function of the characteristic life time of the Ball/Test board Solder joint.

\section{Conclusion}

This paper focused on the application of metamodel based probabilistic method for the reliability assessment of tape based CSP. The finite element analysis is performed to predict the reliability performance of CSP through accelerated temperature cycling solder joint characteristic fatigue life. To avoid tedious computation, kriging metamodel is used. Then, metamodel based probabilistic method, which combine the MCS and the constructed metamodel, was presented and applied to take into account the uncertainties in the reliability analysis of CSP.

\section{References}

[1] B. A. Zahn, Impact of ball via configurations on solder joint reliability in tape-based CSP, Electronic Components and Technology Conference (2002). Proceedings, pages 1475-1483. IEEE.

[2] H. Hamdani, B. Radi, and Z El Hami, Métamodélisation pour une conception robuste des systèmes mécatroniques, Incertitudes et fiabilité des systèmes multiphysiques, 210.

[3] A. El Hami, and P. Pougnet, Embedded Mechatronic Systems, Volume 1, Elsevier, Amsterdam, Netherlands, 2015.

[4] JEDEC STANDARD, Temperature Cycling, JESD22-A104-B. JEDEC Solid state technology association, July 2000. 